instead of
writing or
erasing DWs,
which typically
requires the
application of
a high voltage
to induce
polarization
reversal, we
modulate the
conductivity of
existing DWs

$\Rightarrow$ FERROELECTRIC MATERIALS

\section{Bending walls}

Domain wall (DW) electronics aims to exploit conducting DWs in ferroelectric materials to realize next-generation nano-scale devices. Ferroelectric DWs can be created, erased and moved using electrical fields, but for practical applications it is essential to find simple ways of controlling how they transport charge. Now, Alexei Gruverman and colleagues demonstrate electrical tuning of existing ferroelectric DWs, obtaining a modulation of their conductivity by almost two orders of magnitude, as they report in Advanced Materials.

"Throughout the history of ferroelectric research, DWs were considered as objects of little importance, but with the advent of electron and scanning probe microscopy it became possible to distinguish their properties from those of the surrounding domains," comments Gruverman. "About 10 years ago, it was discovered that DWs in insulating ferroelectrics could behave as good electrical conductors: this discovery has evolved into a new electronic device paradigm, in which the device functionality is determined not by the domains, but by the presence or absence of DWs."

In the new experiments, a thin film of $\mathrm{LiNbO}_{3}-$ a well-known ferroelectric material - was initially prepared to show an upward polarization, then a piezoresponse force microscope was used to apply voltage pulses and stimulate the nucleation of downward-pointing domains, leading to the formation of DWs. "Cross-sectional transmission electron microscopy measurements performed by our colleagues in Limerick and Belfast showed that the DWs deviate from the polar axis direction by almost $20^{\circ}$, which is very unusual, because this deviation makes the DWs electrically charged, and charged DWs are presumably unstable," says Gruverman. "We are still not sure about the reason for their stability." The probe was then used to apply voltage pulses well below the voltages needed to create or move DWs, which resulted in a continuous and reversible tuning of the DWs between a conducting and an insulating state. Local control was also demonstrated, with the induction of insulating segments in conducting walls or vice versa.

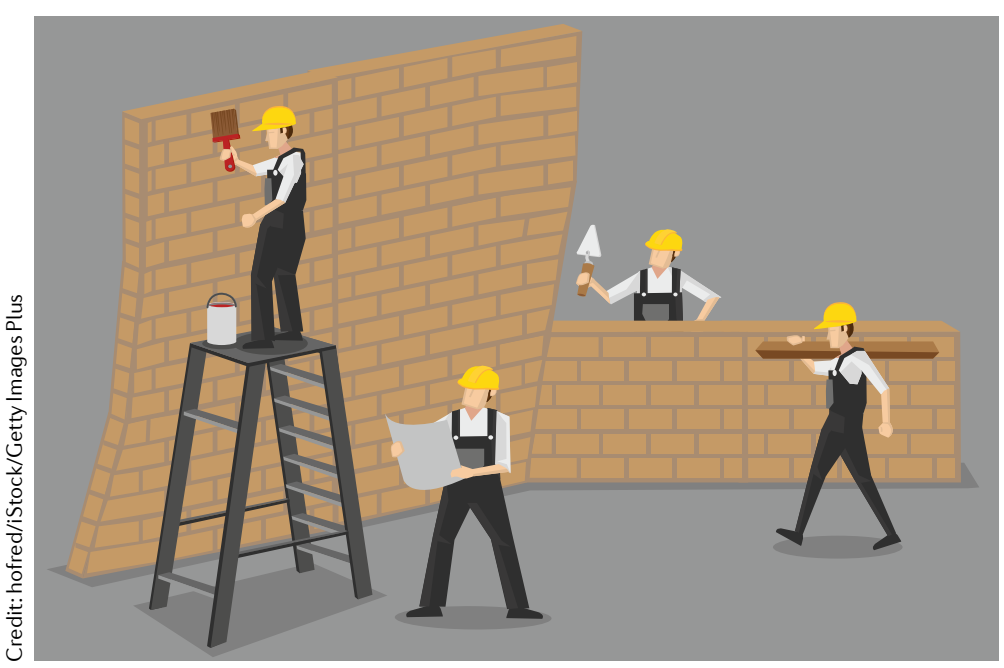

Surprisingly, conductivity changes in the DWs do not influence the domain configuration although, when a DW changes its conductive state, it also slightly shifts. "The novelty of our results is that instead of writing or erasing DWs, which typically requires the application of a high voltage to induce polarization reversal, we modulate the conductivity of existing DWs, achieving the same type of functionality with a much lower voltage," explains Gruverman.

Transmission electron microscopy measurements of cross sections of the samples revealed the mechanism behind the writing process: the DW conductivity depends on the bending of the DWs near the film surface, which influences the charge carrier density and is in turn controlled by the applied voltage pulses. This picture is also supported by a model developed by Gruverman's collaborators at Penn State University.

The DW conductivity as a function of pulse amplitude also shows a hysteretic behaviour. Positive voltage pulses enhance the DW conductivity, and negative voltage pulses suppress it, with a cumulative effect cycle after cycle. This historydependent behaviour makes the DWs promising candidates for potential memristive applications as multilevel logic elements.

The conductive DWs in $\mathrm{LiNbO}_{3}$ thus hold promise for applications in nanoelectronics and memristive devices. "Based on these findings, we plan to develop prototype devices to explore mechanisms of both resistance control, writing and erasing DWs and modulating the conductivity of existing DWs," concludes Gruverman. "We will compare the benefits and drawbacks of each approach."

Giulia Pacchioni

ORIGINAL ARTICLE Lu, $\mathrm{H}$. et al. Electrical tunability of domain wall conductivity in $\mathrm{LiNbO}_{3}$ thin films. Adv. Mater. https://doi.org/10.1002/ adma.201902890 (2019) 